\title{
Тонкопленочные барорезисторы на основе твердых растворов $\mathrm{Sm}_{1-x} \mathrm{Gd}_{x} \mathrm{~S}$
}

\author{
(ㅇ В.В. Каминский ${ }^{1}$, С.М. Соловьев ${ }^{1, \uparrow}$, Н.Н. Степанов ${ }^{1}$, Г.А. Каменская ${ }^{1}$ Г.Д. Хавров ${ }^{1,2}$, С.Е. Александров ${ }^{2}$ \\ ${ }^{1}$ Физико-технический институт им. А.Ф. Иоффре Российской академии наук, \\ 194021 Санкт-Петербург, Россия \\ ${ }^{2}$ Санкт-Петербургский политехнический университет Петра Великого, \\ 195251 Санкт-Петербург, Россия \\ ๑ E-mail: serge.soloviev@mail.ioffe.ru
}

Поступила в Редакцию 1 июня 2020 г.

В окончательной редакции 7 сентября 2020 г.

Принята к публикации 14 сентября 2020 г.

Исследовано влияние концентрации $\mathrm{Gd}$ на температурный и барический коэффициенты электросопротивления тонких поликристаллических пленок твердых растворов системы $\mathrm{Sm}_{1-x} \mathrm{Gd}_{x} \mathrm{~S}$, где $x=0,0.05$, $0.1,0.2,0.33$ и 0.5. Пленки изготовлены методом взрывного испарения в вакууме порошков исходных соединений и седиментацией последних из газовой фазы на стеклянные подложки. Получены зависимости температурного $\alpha$ и барического $\beta$ коэффициентов электросопротивления, а также их отношения $\gamma$ от концентрации $x \mathrm{Gd}$ в системе твердых растворов $\mathrm{SmS}-\mathrm{GdS}$, на основании которых определены оптимальные составы для изготовления тонкопленочных тензо- и барорезисторов.

Ключевые слова: тензо- и барорезистор, содержание $\mathrm{Sm}$ и $\mathrm{Gd}$, моносульфид самария, температурный и барический коэффициенты электросопротивления.

DOI: 10.21883/FTP.2021.01.50380.9440

\section{1. Введение}

В течение ряда лет в ФТИ им. Иоффе РАН проводились научно-исследовательские работы по изучению и созданию тензочувствительных материалов на основе моносульфида самария ( $\mathrm{SmS})$. В результате проведенных экспериментов было установлено, что некоторые составы системы твердых растворов (TP) SmS-GdS могут оказаться перспективными для изготовления на их основе электрических полупроводниковых датчиков механических величин - тензо- и барорезисторов. В [1] был предложен тензочувствительный полупроводниковый материал, представляющий собой $\mathrm{TP} \approx 0.6$ мол\% $\mathrm{Gd}$ в $\mathrm{Sm}-\mathrm{Sm}_{0.994} \mathrm{Gd}_{0.006} \mathrm{~S}$, который имел температурный коэффициент сопротивления (ТКС) $\alpha=2 \cdot 10^{-5} \mathrm{~K}^{-1}$, т.е. в $\approx 200$ раз меньший, чем таковой у чистого SmS, при практически равном по величине коэффициенте тензочувствительности $K=\Delta R /(R \cdot \varepsilon)=138$, где $\Delta R / R$ относительное приращение электросопротивления материала под действием деформации $\varepsilon$. К существенному недостатку указанного материала следует отнести его низкое удельное сопротивление $\rho \approx 8.2 \cdot 10^{-3} \mathrm{OM} \cdot \mathrm{cm}$, препятствующее возможности его применения в качестве чувствительного элемента монокристаллических датчиков высокого давления.

В настоящей работе представлены результаты исследования температурных (в диапазоне $20-150^{\circ} \mathrm{C}$ ) и барических (до 0.6 ГПа при $20^{\circ} \mathrm{C}$ ) зависимостей тонких поликристаллических пленок системы $\mathrm{TP} \mathrm{SmS}-\mathrm{GdS}$, проведенного с целью изучения возможности разработки на их основе высоко тензо- и барочувствительных ма- териалов для пленочных электрических датчиков гидростатического давления (барорезисторов) с приемлемым для практического использования ТКС.

\section{2. Методика экспериментов}

Тонкие поликристаллические пленки ТP $\mathrm{Sm}_{1-x} \mathrm{Gd}_{x} \mathrm{~S}$, где $x=0,0.05,0.1,0.2,0.33$ и 0.5 , были получены методом взрывного (дискретного) испарения порошков исходных соединений в вакууме и седиментацией последних из газовой фазы на разогретые до $400^{\circ} \mathrm{C}$ стеклянные подложки [2]. На сформированные на подложках поликристаллические пленки синтезируемых материалов in situ напылялись также никелевые контакты для последующих электрических измерений. В результате завершения технологического цикла в установке вакуумного напыления на 4-х стеклянных подложках (носителях) были получены 144 пленки с электрическими контактами, которые представляли собой заготовки тензои барорезисторов. Извлеченные из установки подложки разрезались алмазными дисками на отдельные пленки на носителях, к никелевым контактам подпаивались медные измерительные провода, и приготовленные таким образом тензо- и барорезисторы поступали на предварительный контроль их электросопротивления. Далее материал пленок тензо- и барорезисторов проходил рентгеноструктурный и фазовый анализы. Результаты исследований, полученные рентгеновскими методами, как то: постоянные решеток твердых растворов, области когерентного рассеяния рентгеновских лучей (ОКР), фазовый состав пленок оказались полностью идентичными 
таковым, полученным ранее в [3] для указанных ТР. Толщины пленок также, как и в [3], составляли $\approx 0.5$ мкм.

Исследования температурных зависимостей электросопротивления $R$ пленочных тензо- и барорезисторов проводились в интервале температур $20-150^{\circ} \mathrm{C}$ в воздушной атмосфере в термостате. Испытуемые образцы размещались в центре термостата в заполненном микропорошком корунда (фракции 3/5 мкм) алундовом контейнере для создания в нем безградиентного теплового поля в процессе электрических измерений, проводимых на постоянном токе.

На основании полученных зависимостей $R(T)$ образцов различных составов рассчитывались их ТКС $\alpha(T)=\partial(\ln R) / \partial T$ для температурного интервала $20-150^{\circ} \mathrm{C}$. Температура образцов, размещенных в контейнере, измерялась термопарой медь-константан, дополнительно общая температура в термостате контролировалась ртутным термометром.

Для исследования барических зависимостей электросопротивления тензо- и барорезисторов при $T=300 \mathrm{~K}$ применялась малогабаритная поршневая камера высокого гидростатического давления (КВД) до 1.8 ГПа [4]. Образы для исследований размещались на стойке в КВД. В качестве среды, передающей гидростатическое давление $P$ на испытуемые материалы, применялась полиэтилсилоксановая жидкость ПЭС-5 [5]. Измерения $R(P)$ до 0.6 ГПа проводились на постоянном токе при $T=300 \mathrm{~K}$. Давление в камере измерялось манганиновым датчиком высокого давления. На основании полученных зависимостей $R(P)$ рассчитывались БКС $\beta=\partial(\ln R) / \partial P$ пленок, рассматриваемых в настоящей статье ТP.

\section{3. Результаты и их обсуждение}

В процессе исследовательской работы синтезированы тонкие пленки $\mathrm{Sm}_{1-x} \mathrm{Gd}_{x} \mathrm{~S}$ перечисленных выше составов, и проведена их стандартная паспортизация.

Для указанных пленок построены зависимости ТКС $\alpha$ и БКС $\beta$ от количества допирующей $\mathrm{SmS}$ компоненты $x$ - Gd. Полученные данные представлены на рис. 1.

Анализ полученных результатов начнем с рассмотрения зависимости ТКС $\alpha$ от количества $\mathrm{Gd} x$ в твердом растворе $\mathrm{Sm}_{1-x} \mathrm{Gd}_{x} \mathrm{~S}$. Обращает на себя внимание тот факт, что при комнатной температуре ТКС $\alpha(x)$ достаточно быстро убывает по модулю с ростом $x$, однако остается отрицательным (что характерно для невырожденных и слабо вырожденных полупроводников) даже при переходе через границу фаз $(C)$ : полупроводниковой (I) и состояния промежуточной валентности (СПВ) катионов $\mathrm{Sm}(\mathrm{II})^{1}$. Объяснение этого нетривиального факта сохранения отрицательных значений ТКС заключается в предположении возможности перехода одного

\footnotetext{
${ }^{1} \mathrm{C}$ проблемой СПВ ионов $\mathrm{Sm}$ в $\mathrm{SmS}$ и TP на его основе с Gd можно ознакомиться в [6].
}

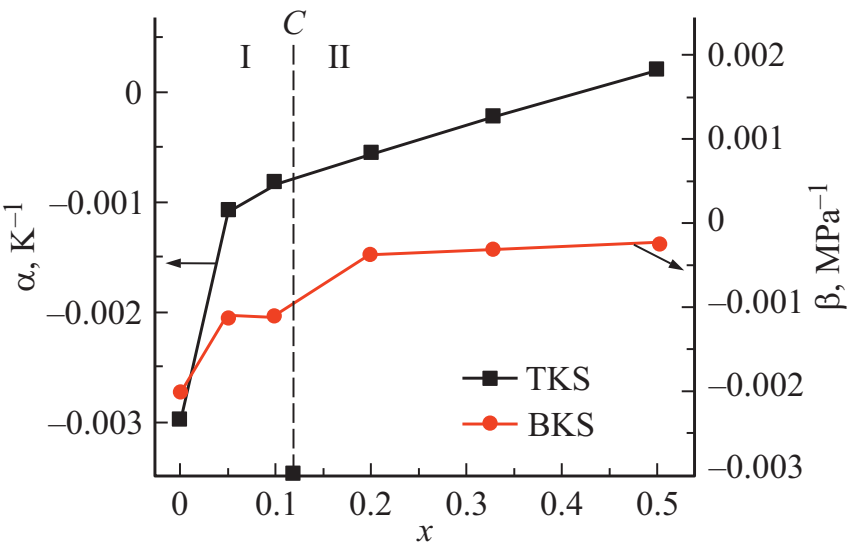

Рис. 1. Зависимость ТКС и БКС тонких пленок твердого раствора $\mathrm{Sm}_{1-x} \mathrm{Gd}_{x} \mathrm{~S}$ от количества допирующей компоненты $x$ - Gd: I - область полупроводниковой (П/П) фазы твердых растворов $\mathrm{SmS}-\mathrm{GdS}$ при нормальных условиях; II область фазы состояния промежуточной валентности (СПВ) катионов $\mathrm{Sm}$ в твердых растворах $\mathrm{SmS}-\mathrm{GdS}$ при нормальных условиях; $C$ - граница фаз.

из $4 f$-электронов катиона $\mathrm{Sm}$ на экситоноподобный (локализованный) $5 d$-уровень в результате фазового превращения, запускаемого увеличением концентрации $x$ катионов $\mathrm{Gd}$ в твердых растворах: выше 15 мол\% в объемных образцах и 12\% - в пленках [3]. Электроны, заполняющие локализованные $5 d$-состояния, под действием тепла забрасываются в зону проводимости, обеспечивая тем самым активационный характер электропереноса. С ростом концентрации Gd $x$ в системе твердых растворов $\mathrm{SmS}-\mathrm{GdS}$ наблюдаются: во-первых, уменьшение параметра решетки, особенно резкое в области $x \leq 12 \%$ в пленках и $\leq 15 \%$ в объемных образцах, так называемый „химический коллапс“, и, во-вторых, рост концентрации свободных электронов, поскольку каждый катион $\mathrm{Gd}$ поставляет $1 e^{-}$в зону проводимости. В результате совокупного действия описываемых процессов часть электронов уходит с локализованных $5 d$-состояний катионов Sm в зону проводимости, дополнительно увеличивая степень ее заполнения и повышая тем самым уровень химического потенциала в материале. При достижении определенной концентрации свободных электронов в ТP системы $\mathrm{SmS}-\mathrm{GdS}$ устанавливается металлический характер проводимости, наблюдаемый в соединении $\mathrm{Sm}_{0.5} \mathrm{Gd}_{0.5} \mathrm{~S}$ при $T=300 \mathrm{~K}$.

Характер зависимости БКС $\beta$ от концентрации $x \mathrm{Gd}$ в $\mathrm{Sm}_{1-x} \mathrm{Gd}_{x} \mathrm{~S}$ аналогичен таковому для ТКС $\alpha(x)$ с тем небольшим отличием, что функция $\beta(x)$ не выходит в область положительных значений для всех исследованных составов $\mathrm{Sm}_{1-x} \mathrm{Gd}_{x} \mathrm{~S}$. Этот факт объясняется изменением структуры электронного спектра изучаемых объектов под действием всестороннего сжатия. При этом уменьшается глубина залегания локализованных $5 d$-состояний ионов $\mathrm{Sm}$, что способствует сохранению активационного механизма процесса электропереноса в ТР. 


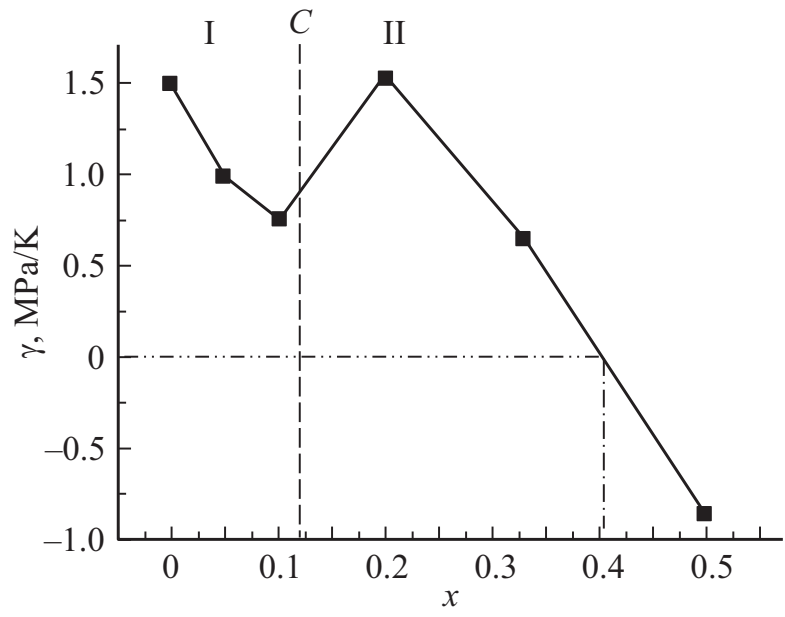

Рис. 2. Зависимость параметра $\gamma$ от количества допирующей компоненты $x$ в твердом растворе $\mathrm{Sm}_{1-x} \mathrm{Gd}_{x} \mathrm{~S}$ : I - область полупроводниковой (П/П) фазы системы твердых растворов $\mathrm{SmS}-\mathrm{GdS}$ при нормальных условиях; II - область фазы состояния промежуточной валентности (СПВ) катионов Sm в твердых растворах $\mathrm{SmS}-\mathrm{GdS}$ при нормальных условиях; $C$ - граница фаз; штрихпунктирная линия выделяет состав ТР, имеющий ТКС $\alpha=0$.

Полученные в настоящей работе зависимости температурного $\alpha$ и барического $\beta$ коэффициентов электросопротивления от концентрации $x \mathrm{Gd}$ в системе $\mathrm{Sm}_{1-x} \mathrm{Gd}_{x} \mathrm{~S}$ предоставляют возможность определить составы, оптимальные для изготовления чувствительных элементов тонкопленочных тензо- и барорезисторов. С целью решения задачи поиска таких составов для каждой из исследованных пленок ТP $\mathrm{Sm}_{1-x} \mathrm{Gd}_{x} \mathrm{~S}$ был рассчитан параметр $\gamma=\alpha / \beta$ и построен график $\gamma(x)$, который представлен на рис. 2. Обращает на себя внимание немонотонный характер поведения зависимости $\gamma$ от $x$. В области I полупроводниковых фаз величина $\gamma$ имеет минимум для состава с $x=0.1$. Поскольку параметр $\gamma$ численно равен кажущемуся приращению давления в окружающей пленку среде при изменении температуры последней на $1^{\circ}$, то TP $\mathrm{Sm}_{0.9} \mathrm{Gd}_{0.1} \mathrm{~S}$ представляется наиболее перспективным для изготовления на его основе чувствительных к давлению и деформациям пленочных полупроводниковых резистивных датчиков ${ }^{2}$. Из приведенной на рис. 2 зависимости $\gamma(x)$ следует, что пленка с составом $\mathrm{Sm}_{0.9} \mathrm{Gd}_{0.1} \mathrm{~S}$ обладает достаточно высоким БКС в сочетании с низким ТКС. Величина параметра $\gamma \quad$ у $\quad \mathrm{Sm}_{0.9} \mathrm{Gd}_{0.1} \mathrm{~S}$ близка к таковой у $\mathrm{TP}$ $\mathrm{Sm}_{0.65} \mathrm{Gd}_{0.35} \mathrm{~S}$ с СПВ катинов $\mathrm{Sm}$ и меньше, чем у полупроводниковой пленки $\mathrm{SmS}$ в $\sim 2$ раза. Следовательно, оптимальным составом для изготовления тонкопленочных барорезисторов на основе системы $\mathrm{SmS}-\mathrm{GdS}$ для не очень высоких гидростатических давлений $(\leq 0.4$ ГПа

\footnotetext{
2 Строго говоря, речь может идти только о некоторой узкой области составов, близких к указанному. Воспроизвести требуемый состав в пленках от партии к партии достаточно сложно; отклонение от исходной химической формулы может составлять до $10 \%$ [3].
}

согласно фазовой диаграмме состояний [6]) является ТР $\mathrm{Sm}_{0.9} \mathrm{Gd}_{0.1} \mathrm{~S}$.

Пленки твердых растворов из области II фазовых состояний обладают невысокой чувствительностью к давлению (см. рис. 1), но в то же время и низкими значениями ТКС. Помимо этого, они не претерпевают фазовых превращений под действием всестороннего сжатия и, следовательно, могут представлять интерес как материалы для разработки барорезисторов на область высоких давлений (скажем, превышающих 0.65 ГПа давление фазового перехода в $\mathrm{SmS}$ [6]).

Из рис. 2 следует, что состав $\mathrm{Sm}_{1-x} \mathrm{Gd}_{x} \mathrm{~S}$ с $x \approx 0.4$ имеет ТКС $\alpha=0$ и в силу данного обстоятельства представляется перспективным для разработки на его основе независимого от температуры барорезистора.

\section{4. Заключение}

На основании полученных в настоящей работе результатов определена область составов, близких к $\mathrm{Sm}_{0.9} \mathrm{Gd}_{0.1} \mathrm{~S}$, имеющих высокие значения БКС при достаточно слабом влиянии температуры на их удельное электросопротивление. Указано на возможность использования тонких поликристаллических пленок состава $\mathrm{Sm}_{0.9} \mathrm{Gd}_{0.1} \mathrm{~S}$ в качестве чувствительного элемента тензои барорезисторов.

Показано, что состав $\mathrm{Sm}_{0.6} \mathrm{Gd}_{0.4} \mathrm{~S}$ должен обладать близким к нулю ТКС и, следовательно, может быть использован в качестве чувствительного элемента в пленочных датчиках высокого давления.

\section{Конфликт интересов}

Авторы заявляют, что у них нет конфликта интересов.

\section{Список литературы}

[1] В.В. Каминский, М.В. Романова, А.В. Голубков, В.М. Сергеева, И.А. Смирнов. А. с. № 1407132 . Приоритет изобретения 16.04.1985.

[2] В.В. Каминский, Н.М. Володин, С.М. Соловьев, Ю.Н. Мишин, Н.В. Шаренкова. Вестн. „НПО им. С.А. Лавочкина“, 2, 26 (2013).

[3] В.В. Каминский, С.М. Соловьев, Г.Д. Хавров, Н.В. Шаренкова. ФТП, 52 (1), 45 (2018).

[4] Е.С. Ицкевич. ПТЭ, 3, 6 (1999).

[5] А.С. Кириченко, А.В. Корнилов, В.М. Пудалов. ПТЭ, 6, 121 (2005).

[6] В.С. Оскотский, И.А. Смирнов. В кн.: Редкоземельные полупроводники, отв. ред. В.П. Жузе, И.А. Смирнов (Л., Наука. Ленингр. отд-ние, 1977) с. 105.

Редактор Г.А. Оганесян 
Thin-film baroresistors based on solid solutions $\mathbf{S m}_{1-x} \mathbf{G d}_{x} \mathbf{S}$

V.V. Kaminsky ${ }^{1}$, S.M. Soloviev ${ }^{1}$, N.N. Stepanov ${ }^{1}$, G.A. Kamenskaja ${ }^{1}$, G.D. Khavrov ${ }^{1,2}$, S.E. Alexandrov ${ }^{2}$

${ }^{1}$ loffe Institute,

194021 St. Petersburg, Russia

${ }^{2}$ Peter the Great St. Petersburg Polytechnic University, 195251 St. Petersburg, Russia

Abstract The effect of Gd concentration on the temperature and baric coefficient of electrical resistance of thin polycrystalline films of solid solutions of the $\mathrm{Sm}_{1-x} \mathrm{Gd}_{x} \mathrm{~S}$ system, where $x=0,0.05$, $0.1,0.2,0.33$ and 0.5 . Films are made by explosive evaporation in vacuum of powders of initial compounds and sedimentation of the latter from the gas phase on glass substrates. The dependences of the temperature $\alpha$ and baric $\beta$ coefficients of electrical resistance, as well as their ratio $\gamma$ on the concentration $x \mathrm{Gd}$ in the system of solid solutions of $\mathrm{SmS}-\mathrm{GdS}$, are obtained, on the basis of which the optimal compositions for the manufacture of thin - film tensor and baroresistors are determined. 